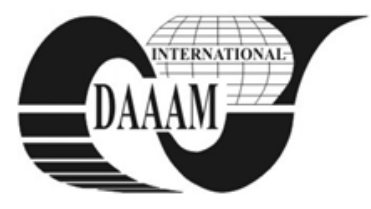

Annals of DAAAM for 2011 \& Proceedings of the 22nd International DAAAM Symposium, Volume 22, No. 1, ISSN 1726-9679 ISBN 978-3-901509-83-4, Editor B. Katalinic, Published by DAAAM International, Vienna, Austria, EU, 2011 Make Harmony between Technology and Nature, and Your Mind will Fly Free as a Bird Annals \& Proceedings of DAAAM International 2011

\title{
TRANSMISSION OF THE CALORIC EFFECTS BY THE OSCILANTING WAVES
}

\author{
BAL, C[armen] \& BAL, N[icolaie]
}

\begin{abstract}
The paper presents some aspects about the caloric effect created by oscillating waves. We present some theoretical and experimental aspects of the caloric effect and transmission by oscillating waves.

Key words: sonic flow, sonic pressure, sonic capacity, friction coefficient, sonic induction and sonic inertia
\end{abstract}

\section{INTRODUCTION}

The sonic actions permit the best combination of facilities offered by the processing of electrical signals (reduces energy) with sonically actions of great power and efficiency, which give the possibility of eliminating the biggest parts of a classical hydraulic system (hydraulic reservoir, flow-adjustment valve), leading to an action which combines the opportunities offered by the processing of the signals of low energy and the compact sonic actions, with high efficiency, with reduces volume, i. e. it is very economic. Here it has to be mentioned the fact that, this theory is a particular case of power transmission through movement.

By using a precinct for each type (C) the pipe could be closed partially or totally. So, it will be possible to fait at one end or at one intermediate point, as apparatus for the partial use of wave energy, and the rotated crack $(m)$ will produce work only if the energy is efficiently used. It could be supposed that the pipe is closed in point ( $r$ ) at an equal distance with a full multiple, $n$, of wave length, beginning from the wave generator (g) and that there are branches $\mathrm{b}, \mathrm{c}, \mathrm{d}$ at distance of $\frac{\lambda}{2} ; \frac{3}{4} \lambda$ and $\lambda$, figure 1 .

We know, from the analysed cases above, that if the tap (r) is closed and the tap $\left(r_{3}\right)$ is open, leading to engine $\left(m_{3}\right)$, which is spilling with a synchronized speed, it will be able to absorb the energy introduced in liquid by whole the generator $(\mathrm{g})$.

If all taps are closed, in the pipe will appear stationary waves, having the maximum of variation of pressure at the end of (r) and at $\frac{\lambda}{2}$ (though in b point). In these points the flow will be always zero, and the pressure will alternate between the extreme values determined by the (C) capacity. At the distances of $\frac{\lambda}{4}$ and $\frac{3}{4} \lambda$ in points (a) and (c), the flow will alternate between the extreme values, but the pressure variation will be zero. In this case, the pressure and maximum movement points not move along the pipe, but are fix in position, and theoretically, no-energy don't flows from the generator. However, at maximum movement points, the pressure variation is null, and the points of maximum variation pressure, it will not produce any fluid movement:

a) It is clear that if the tap $\left(r_{1}\right)$, which leads directly to the engine $\mathrm{m}_{1}$, is open, it will rotate with a synchronic speed, being able to absorb the whole energy communicated to the line. So, the stationary semi wave between $(\mathrm{g})$ and (b) disappears, being replaced by a progressive wave, as long as between (b) and the need of the line (r), the stationary wave will persist.

b) If the tap $\left(\mathrm{r}_{2}\right)$, which will lead to the engine $\left(\mathrm{m}_{2}\right)$ (settled at $\frac{3}{4} \lambda$ ) is open, all other taps being closed, now in (c) point, the pressure variation is always zero, none energy will be absorbed by the engine, and the stationary wave will persist in the entire length of the pipe.

c) If the engine is connected at the intermediate point, a part from the energy will be absorbed by the engine, or stationary wave will persist with reduced amplitude between the generator $(\mathrm{g})$ and engine, the liquid state between it could be considered as result of two overlapped waves: one stationary wave and one progressive wave.

d) Let's assume that, in this case, the engine $\left(\mathrm{m}_{3}\right)$ is not able to absorb the whole energy communicated to the line by the generator $(\mathrm{G})$. We will have then overlapped in the pipes a system of stationary waves and a system of mobile waves along the pipe, though there will not be any point in the pipe where the variation of the pressure will be permanently zero. So, a connected engine at any point of pipe will be an able to absorb and use an action from the energy communicated to the line.

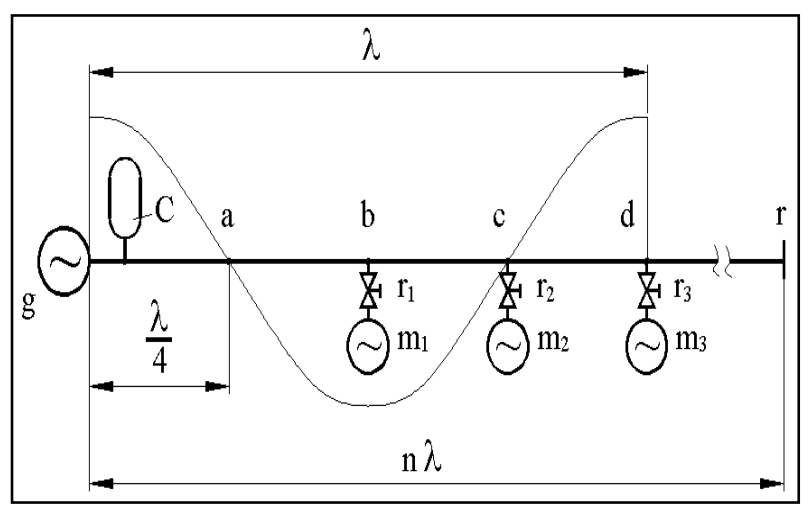

Fig. 1. Oscillanting system

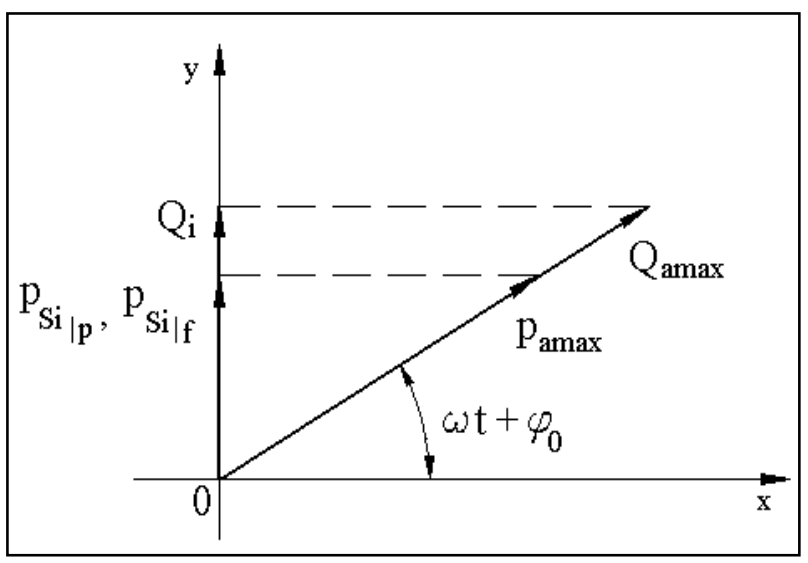

Fig. 2. The variation of the pressure and the flow 


\section{THE CALORIC \\ EFFECTS OSCILANTINGS WAVES}

The differential equation for one pipe to taking into account inertia and liquid capacity, the equation can be:

$$
\begin{aligned}
& \frac{d \bar{Q}}{d x}=j \cdot \omega \cdot \bar{C}_{s} \cdot \bar{p}_{a_{\max }} \\
& \frac{d \overline{p_{a_{\max }}}}{d x}=j \cdot \omega \cdot \bar{L}_{s} \cdot \bar{Q}_{a_{\max }}
\end{aligned}
$$

The instantaneous flow has one simple harmonic variation, of the form:

$$
Q_{i}=Q_{a \max } \cdot \sin \left(\omega t+\varphi_{0}\right)
$$

In relation $\Delta p_{f i}=R_{f} \cdot Q_{i}$ and $Q_{i}=C_{p} \cdot \Delta p_{s i}$ the friction coefficient $\mathrm{R}_{\mathrm{f}}$ and the perditance $\mathrm{C}_{\mathrm{p}}$, are constants.

The relationships above can be writing:

$$
\begin{gathered}
\Delta p_{f i}=R_{f} \cdot Q_{i} \\
\Delta p_{s i}=R_{p} \cdot Q_{i} \\
R_{p}=\frac{1}{C_{p}}
\end{gathered}
$$

where:

Value $i$ refers to the instantaneous values.

If the flow is sinusoidal we have the relation:

$$
Q_{a \max }=C_{p} \cdot p_{a \max }
$$

For the friction we have the relation:

$$
p_{a \max }=C_{f} \cdot Q_{a \max }
$$

In phase with flow, Figure 2, where $R_{f}=C_{f}$ and is the friction coefficient.

We propose to observe the friction effects due to account of the variation of the different elements. We need to calculate this friction and the importance for the caloric effects that can developed.

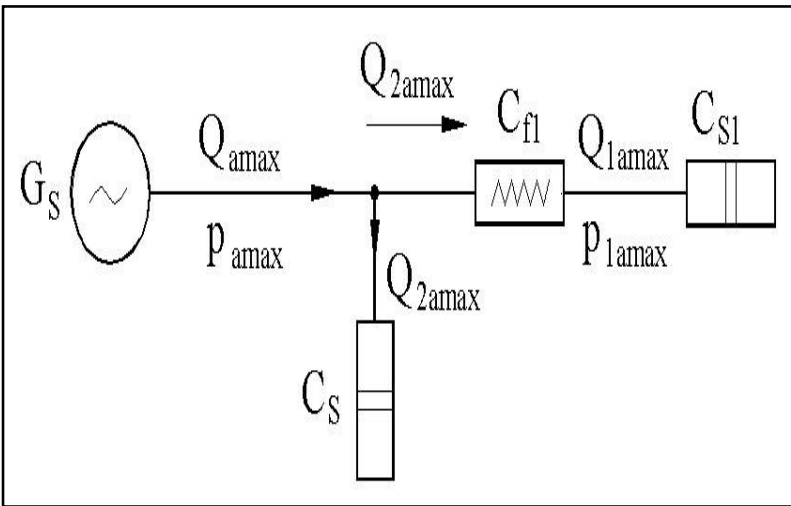

Fig. 3. The oscilanting circuit

To high light the caloric effects in a sonic circuit, we consider the circuit form by the condenser and the friction resistance (Figure 3).

If we take into account the volume of the condenser we see the variation of the friction resistance, function of this variation.
In figure 4 we observe that at constant angle speed and constant volume of a condenser we see the upper to the value of the friction coefficient increase

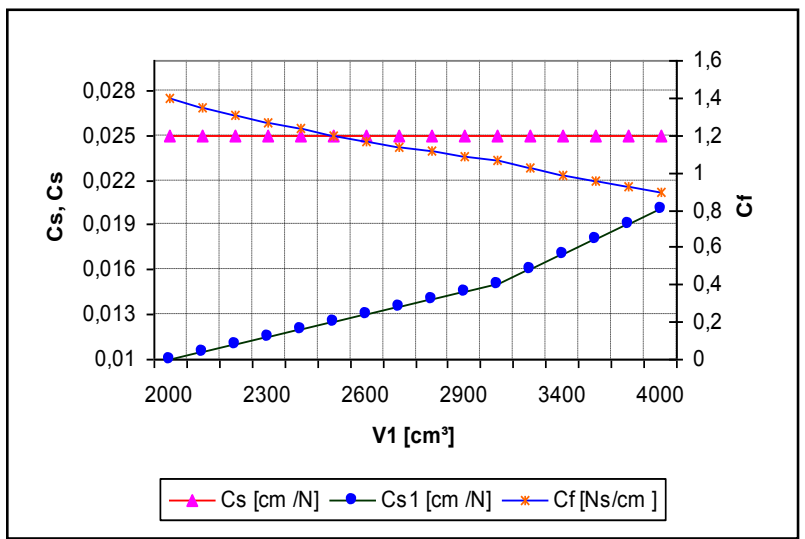

Fig. 4. The variation of the volume about the friction coefficient

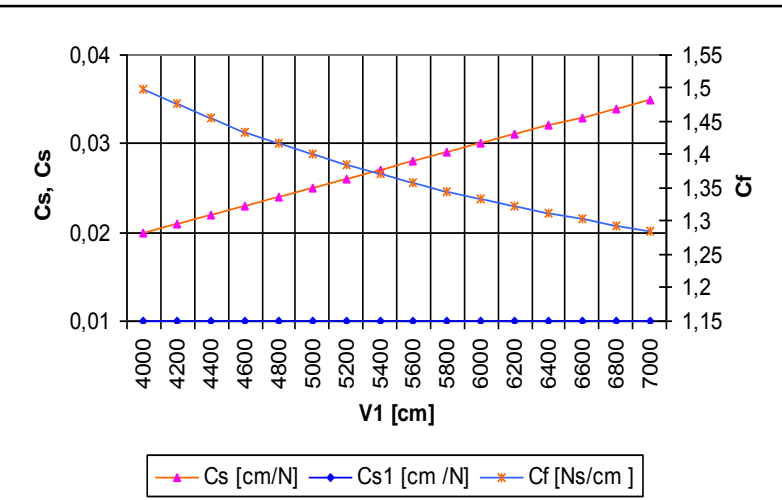

Fig. 5. The variation of the volume about the friction coefficient

If we take into account the volume of the condenser we see the variation of the friction resistance, function of this variation. In figure 5 we observe that at constant angle speed and constant volume of condenser, we see the upper to the value of the friction coefficient increase.

\section{CONCLUSION}

1. If we have the variation of the volume of the second condenser, we observe the increase of the value of the friction coefficient. In this case the angle speed is constant.

2. In the second case we see the variation of the volume of the first condenser; in the graphic we see increase of to the friction coefficient. The angle speed is also constant.

3 . In this case highlights the emergence of caloric effect in friction resistance.

\section{REFERENCES}

Constantinescu, G., (1985), The theory of the sonicity, București, Editura Academiei

Carmen Bal, (2007), Caloric effect in the circuits by harmonic flow, Cluj Napoca, Ed. ALMA MATER, ISBN 978-9737898-75-3

Carmen Bal, (2006), Research and contributions about the drive systems with the harmonic flow, the doctoral thesis Technical University of Cluj Napoca

Pop I. Ioan, Carmen Bal, Marcu Lucian ș.a., (2007), The sonicity applications. Experimental results, Iași, Ed. Performantica, ISBN 978-973-730-391-2

Pop I. I., (2006), The treaty of the sonicity theory, Iași, Ed. Perfomantica 